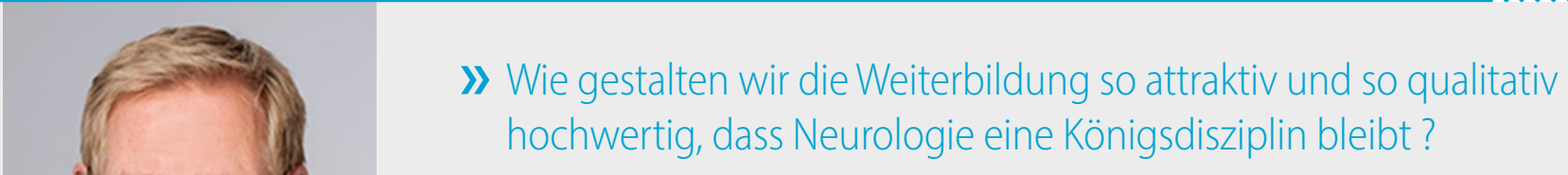

Prof. Dr. Christian Gerloff

Klinik und Poliklinik für Neurologie, Universitätsklinikum Hamburg-Eppendorf

Martinistraße 52, 20246 Hamburg, Deutschland

gerloff@uke.de

\title{
Bergfest
}

Bergfest. So nennt man es wohl, wenn die eine Hälfte hinter einem, die andere vor einem liegt. Nun hatte ich 1 Jahr lang bereits die Ehre und auch die sehr wohl wahrgenommene Verantwortung, als Präsident der DGN (Deutsche Gesellschaft für Neurologie) zu dienen - und ein weiteres, spannendes Jahr der Präsidentschaft liegt vor mir.

Das Jahr 2021 war kein Jahr, in dem man Pläne auf Millimeterpapier zeichnen konnte. Die Coronapandemie bestimmte vieles, aber nicht alles. Pandemie hin oder her, die DGN verfolgte auch davon unabhängig konsequent gesteckte Ziele.

Nachwuchs und Weiterbildung sind ein zentrales Thema. Zentrale Frage ist, wie wir die Weiterbildung so attraktiv und so qualitativ hochwertig gestalten können, dass Neurologie eine Königsdisziplin bleibt. Dazu sind viele gute Zutaten erforderlich: klinische Fertigkeiten und Grundkompetenzen wie Neurophysiologie und -sonologie in all ihren Facetten, und zwar auch dann, wenn die Notfallversorgung in unserem Fach immer zentraler, der Schlaganfall immer dominanter wird. Es geht um eben das, was viele Bewerberinnen und Bewerber schon in den ersten Minuten eines Gesprächs auf die Frage sagen, warum sie Neurologie lernen wollen: Dass man mit den eigenen Händen, mit der kenntnisreichen klinisch-neurologischen Untersuchung gleichsam detektivisch zur Diagnose oder zumindest zu einer präzise eingeengten Auswahl an Differenzialdiagnosen kommt. Von der DGN wurden in diesem Jahr die ersten "skills labs“ erfolgreich organisiert, die Jungen Neurologen auf vielen Ebenen intensiver eingebunden, zunehmend besser standardisierte Facharztrepetitorien angeboten und in dieser Zeitschrift unter Federführung von Prof. Peter Berlit ein reicher Fundus an fallorientierten Materialien für die Facharztweiterbildung zusammengestellt. Mehr wird folgen, und der große Dank gilt schon jetzt all denen, die sich so bereitwillig und intensiv dafür engagierten.

Unser Fach ist inhaltlich immens gewachsen. Das sind grundsätzlich exzellente Nachrichten, v. a. im Hinblick auf die vielen neuen Therapieansätze, die uns heute im Vergleich zu vor 30 Jahren zur Verfügung stehen: 18 zugelassene Immuntherapien gegen multiple Sklerose (MS), 15 Medikamente, die bei Parkinson-Syndromen in unterschiedlicher Weise Dopaminrezeptoren

DGNeurologie $2022 \cdot 5(1): 1-2$

https://doi.org/10.1007/s42451-021-00406-7

Angenommen: 13. Dezember 2021

๑) Springer Medizin Verlag GmbH, ein Teil von Springer Nature 2021 modulieren, Gentherapie bei spinaler Muskelatrophie und vieles mehr; dazu kommen unzählige neue diagnostische Verfahren. Diese Inhalte zu lehren, ist genauso zwingender Bestandteil der Weiterbildung wie die Vermittlung der genuin klinisch-neurologischen Grundkompetenzen. Die simple, aber bedrohliche Feststellung ist, dass wir hier mit den momentan gültigen 48 Monaten Neurologie nicht mehr genügend Zeit haben. Machen wir so weiter, droht unweigerlich ein Kompetenzverlust, mit dem wir uns selbst degradieren. Was wäre ein Facharzt oder eine Fachärztin für Neurologie wert, der oder die sich nach bestandener Prüfung in einem Eskalationsschema der MS-Therapie nicht zurechtfindet, keine Ahnung hat, welche Impfungen vor Beginn einer Immuntherapie zu verabreichen sind, nicht weiß, welche Parkinson-Patienten von der tiefen Hirnstimulation profitieren, oder welche modernen neuroonkologischen oder antikonvulsiven Konzepte heute Anwendung finden. Und ja, auch Gentherapie bei seltenen Erkrankungen ist ein Thema der fachärztlichen Weiterbildung, keine Zusatzqualifikation. Hier herrschen Modernisierungsstau und Handlungsdruck.

Die Modernisierung der Weiterbildung gelingt nur als Teamwork. Die DGN hat daher eine Arbeitsgruppe gegründet, in der wir mit der Deutschen Gesellschaft für Psychiatrie und Psychotherapie, den Berufsverbänden für Neurologie, Psychiatrie und Nervenärzte, den Jungen Neurologen, der Generation Psy, also den jungen Psychiaterinnen und Psychiatern, in einen konstruktiven Dialog eingetreten sind. Wir werden uns dabei eng von der Bundesärztekammer beraten und begleiten lassen. Es ist keine Lösung, sich dieses Dilemma tatenlos anzusehen. Und Alternativen gibt es immer. Die Berufsverbände, das sei an dieser Stelle erwähnt, haben sehr viel für die deutsche Neurologie und Psychiatrie erreicht und sich z. B. frühzeitig in einem Spitzenverband zusammengeschlossen, um auf politischer Ebene in Berlin mehr Schlagkraft zu haben - mit Erfolg.

Ein weiterer, COVID-19-unabhängiger (COVID-19: „coronavirus disease 2019“), Schritt zur Zukunftssicherung der DGN und der Neurologie in Deutschland insgesamt ist die Digitalisierung. Wir haben für die DGN begonnen, ein komplett neues digitales Konzept, den DGN-Channel aufzubauen. Sie dürfen gespannt sein! Unser Ziel ist das "go-live“ Ende 2022. Die Digitalisierung spielt aber auch inhaltlich-fachlich in der Neurologie eine besonders große Rolle. Assistenzsysteme, die basierend auf maschinellem Lernen differenzialdiagnostische Prozesse oder Therapiepfade unterstützen, werden kommen und bieten sich z. B. im Bereich seltener genetischer Erkrankungen, aber auch 
in der neurologischen Intensivmedizin an, immer da, wo riesige Datenmengen Muster aufweisen, die zumindest für weniger Erfahrene kaum noch zu interpretieren sind. In diesem Feld wird es spannende Entwicklungen geben, und die Neurologie muss vorne dabei sein.

Dass die DGN im Hinblick auf die COVID-19-Pandemie sehr aktiv war, dürfte Ihnen allen nicht entgangen sein: Journal Club, Leitlinien, Mitarbeit im Pandemierat der Bundesärztekammer, nationale Studie zur impfassoziierten Sinus- und zerebralen Venenthrombose, Unterstützung des Registers „Lean European Open Survey on SARS-CoV-2 infected patients" (LEOSS), das auf eine Initiative der Jungen Neurologen zurückgeht und vieles mehr. An dieser Stelle möchte ich allen, die sich als Neurologinnen und Neurologen in der COVID-19-Pandemie engagiert haben und engagieren, herzlich danken. Die nächste Herausforderung an uns wird wohl das Long-COVID-19-Syndrom sein, ein Syndrom, das allerdings nach meinem Dafürhalten einen hochgradig interdisziplinären Ansatz erfordert.

Es war also alles andere als langweilig, dieses erste Jahr der Präsidentschaft. Auch unsere Jahrestagung 2021, bei der ich vie- le von Ihnen lieber in Präsenz persönlich gesehen hätte, war eine sehr erfolgreiche Veranstaltung mit 6700 Teilnehmenden und einem sehr positiven Feedback. Im Rückblick waren wir sehr froh, dass wir uns frühzeitig für ein digitales Konzept und damit solide Planbarkeit entschieden hatten. Die Wucht der vierten Welle und das Durchbrechen der Omikronvariante von SARS-CoV-2 („severe acute respiratory syndrome coronavirus 2”) geben uns Recht.

Umgeben von einem exzellenten multiprofessionellen Team in der Geschäftsstelle der DGN und bewundernswerten Kolleginnen und Kollegen im Präsidium und darüber hinaus freue ich mich nun auf Jahr 2 und wünsche Ihnen viel Freude bei der Lektüre dieser Ausgabe von DGNeurologie.

Christian Gerloff

Interessenkonflikt. C. Gerloff gibt an, dass kein Interessenkonflikt besteht.

\section{Dank an die Gutachterinnen und Gutachter für DGNeurologie 2021}

Für die Qualität und Objektivität der Beiträge sind neben den engagierten Autorinnen und Autoren auch die vielen qualifizierten Gutachterinnen und Gutachter maßgeblich, die im Rahmen des Peer-Review-Prozesses die Manuskripte inhaltlich-wissenschaftlich prüfen und Empfehlungen zur konkreten Verbesserung äußern. Allen Gutachterinnen und Gutachtern, die im vergangenen Jahr Manuskripte für diese Zeitschrift begutachtet haben, danken wir herzlich für die konstruktive und gewissenhafte Arbeit.

Die Redaktion

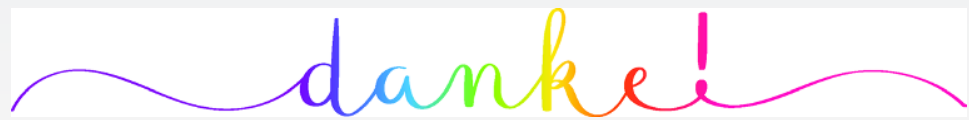

๑ treenabeena / stock.adobe.com 